\title{
Posterior interhemispheric transtentorial approach for resection of a meningioma at the posteromedial tentorial incisura
}

\author{
Sun Liyong, MD, Yuhai Bao, MD, Jiantao Liang, MD, Mingchu Li, MD, and Jian Ren, MD \\ Department of Neurosurgery, Xuanwu Hospital, Capital Medical University, Beijing, China
}

The posterior interhemispheric approach is a versatile approach to access lesions of the pineal region, posterior incisural space, posterior region of third ventricle, and adjacent structures. We demonstrate the case of a 26 -year-old woman with symptoms of increased intracranial pressure and hydrocephalus caused by a meningioma at the posteromedial tentorial incisura. Gross-total removal of the tumor was successfully achieved via a posterior interhemispheric transtentorial approach. The patient reported an immediate and significant symptomatic improvement after surgery. The detailed operative technique and surgical nuances, including the surgical corridor, tentorium incision, tumor dissection and removal are illustrated in this video atlas.

The video can be found here: https://youtu.be/nSNyjQKI7aE.

KEYWORDS posterior interhemispheric transtentorial approach; tentorial incisura; meningioma; resection; video 